\title{
Study on the Flow Structures and Pollutant Dispersion of a Moving Car
}

\author{
Z. Q.Yin ${ }^{1 *}$, J. Z. Lin ${ }^{1}$, K. Zhou ${ }^{2}$
}

${ }^{1}$ China Jiliang University, Hangzhou, 310018, China,

${ }^{2}$ Department of Mechanics, State Key Laboratory of Fluid Power Transmission and Control, Zhejiang University, Hangzhou 310027, China

Email: yinzq@cjlu.edu.cn

\begin{abstract}
The pollutant from vehicular exhaust not only has direct impact on human health, but also constitutes a major fraction of the total pollutant dispersion. At the same time the flow structure plays an important role in the removal of pollutants from the vehicle. So the study on the flow structures and pollutant dispersion is important to improve the air quality in urban areas.

Large eddy simulation (LES) method has been used to calculate the flow structures and vehicular exhaust gas dispersion of the moving car for various conditions. The three-dimensional LES calculates the resolved-scale turbulent motions by directly solving a set of filtered Navier-Stokes equations and models the small subgrid-scale motions. The computational domain is shown in the following figure.
\end{abstract}

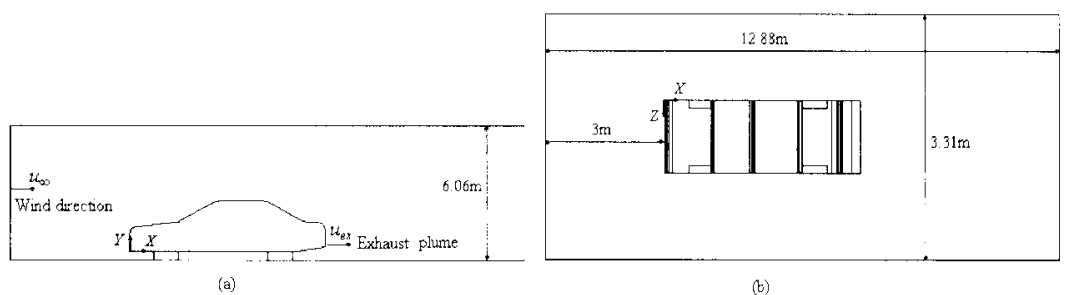

Figure: Computational domain for the CFD simulations. (a) is a side view, (b) is a panel view

The computational results indicate the flow structures behind the moving car wake region are quite comparable with the available experimental results. The flow structure may change with different vehicular exhaust gas speed, wind speed and direction. A larger amount of pollutant is diluted by the high ambient wind speed than by a low ambient wind speed. The ambient wind direction can alter the concentration distribution of vehicular exhaust gas. The results are valuable for us to understand the pollutant dispersion in the near wake region of a vehicle for urban road microenvironments.

\section{REFERENCES}

1. Baker C.J. Flow and dispersion in ground vehicle wakes. J.Fluids \& Struc., 2001;15:1031-1060

2. Dong G, Chan T L. Large eddy simulation of flow structures and pollutant dispersion in the near-wake region of a light-duty diesel vehicle. Atmos. Environ., 2006;40:1104-1116

3. Bearman,P.W. Near wake flows behind two-and three-dimensional bluff bodies. J.Wind Eng. Ind. Aerodyn., 1997;69:33-54 